\title{
Urinary arsenic, pesticides, heavy metals, phthalates, polyaromatic hydrocarbons, and polyfluoroalkyl compounds are associated with sleep troubles in adults: USA NHANES, 2005-2006
}

\author{
Ivy Shiue ${ }^{1,2}$
}

Received: 11 July 2015 / Accepted: 7 November 2016 / Published online: 17 November 2016

(C) The Author(s) 2016. This article is published with open access at Springerlink.com

\begin{abstract}
Links between environmental chemicals and human health have emerged, but the effects on sleep health were less studied. Therefore, the aim of the present study was to investigate the relationships of different sets of environmental chemicals and common sleep troubles in a national and population-based setting. Data were retrieved from the United States National Health and Nutrition Examination Surveys, 2005-2006 including demographics, serum measurements, lifestyle factors, self-reported sleep troubles, and urinary environmental chemical concentrations. Statistical analyses including descriptive statistics, $t$-test, chi-square test, and survey-weighted logistic regression models were performed. Of all 5563 Americans aged 18-85, 2331 (42.0\%) had wake-up at night, 2914 (52.5\%) felt unrested during the day, $740(13.4 \%)$ had leg jerks while sleeping, and 1059 $(19.1 \%)$ had leg cramps for $2+$ times a month. Higher levels of urinary arsenic, phthalates, and polyfluoroalkyl compounds were associated with wake-up at night. Higher levels of urinary 4-tert-octylphenol and polyfluoroalkyl compounds were associated with being unrested during the day. Higher levels of urinary arsenic, polyaromatic hydrocarbons, and polyfluoroalkyl compounds were associated with leg jerks while sleeping. Higher levels of urinary pesticides, heavy metals, phthalates, and polyaromatic hydrocarbons were associated with leg cramps while sleeping. However, there were
\end{abstract}

Editorial responsibility: Philippe Garrigues

Ivy Shiue

ivy.shiue@northumbria.ac.uk

1 Faculty of Health and Life Sciences, Northumbria University, Benton, Newcastle upon Tyne, England NE7 7XA, UK

2 Owens Institute for Behavioral Research, University of Georgia, Athens, GA, USA no significant associations with other environmental chemicals such as parabens, bisphenol A, benzophenone-3, triclosan, perchlorate, nitrate, or thiocyanate. Eliminating arsenic, heavy metals, phthalate, pesticides, polyaromatic hydrocarbons, and polyfluoroalkyl compounds to improve sleep health might be considered while understanding the biological pathway with a longitudinal or experimental approach in future research would be suggested.

Keywords Sleep $\cdot$ Arsenic $\cdot$ Pesticide $\cdot$ Heavy metal · Phthalate $\cdot$ Phenol $\cdot$ Polyfluoroalkyl $\cdot$ Hydrocarbon . Environmental chemical

\section{Introduction}

\section{Evidence before this study}

Links between environmental chemicals and human health in the American adults including hypertension, cardiovascular disease, food allergy, oral health, emotional support, and cognitive function have emerged (Shiue 2015a, b, c, d, 2014a, b, 2013a, b, c), but the effects on sleep health, such as time to fall asleep as one of the indicators, were relatively less studied. Epidemiological studies have shown that the prevalence of sleep disturbances lies between 20 and 30\% and increases with age, particularly in female (Zeitlhofer et al. 2000). Among the American adults, in studying risk contributors for sleep health it was observed that vitamin D level, a hormone that interacts with intranuclear receptors to effect transcriptional changes in many cell types including those in gut, bone, breast, prostate, brain, skeletal muscle, and the immune system (McCarty et al. 2014), could also play an important role in time to fall asleep at bedtime (Shiue 2013d). 


\section{Study aim}

However, it is unclear whether environmental chemicals in addition to sun radiation could influence sleep patterns as well. Following this context, therefore, the aim of the present study was to investigate the relationships of different sets of environmental chemicals and some common sleep troubles in a national and population-based setting using an independent dataset.

\section{Methods}

\section{Study sample}

As described online (more details via http://www.cdc. gov/nchs/nhanes.htm), the United States National Health and Nutrition Examination Surveys (NHANES) has been a national, population-based, multi-year, cross-sectional study since the 1980s. Study samples are representative of the civilian, non-institutionalized US population. Information on demographics, serum measurements, lifestyle factors, sleep pattern (Question: How does it usually take to fall asleep at bedtime? More details via http://wwwn.cdc.gov/Nchs/Nhanes/20052006/SLQ_D.htm) and urinary environmental chemical concentrations was obtained by household interview. In the current analysis, the 2005-2006 cohort as the most recent study cohort with all the required information mentioned above was selected for statistical analysis (more details via http://wwwn.cdc.gov/nchs/nhanes/search/nhanes05_06. aspx). Informed consents were obtained from participating subjects by the NHANES researchers.

\section{Biomonitoring}

Urines were only collected in a representative subsample within 10 days of the household interview, being one third of the whole study cohort (more details via http://www.cdc. gov/nchs/data/nhanes/nhanes_05_06/lab_d.pdf), to measure environmental chemical concentrations in urine among people aged 6 and above (more details via http://www.cdc. gov/nchs/data/nhanes/nhanes_03_04/environmentalhealth_03. pdf). Urine specimens were processed, stored under appropriate frozen $\left(-20^{\circ} \mathrm{C}\right)$ conditions, and shipped to the Division of Environmental Health Laboratory Sciences, National Center for Environmental Health, Centers for Disease Control and Prevention for analysis.

The inductively coupled plasma-mass spectrometry (ICPMS) method (Mulligan et al. 1990) was used to measure the following 12 elements in urine: beryllium $(\mathrm{Be})$, cobalt $(\mathrm{Co})$, molybdenum (Mo), cadmium (Cd), antimony ( $\mathrm{Sb})$, cesium $(\mathrm{Cs})$, tungsten $(\mathrm{W})$, tin $(\mathrm{Sn})$, strontium $(\mathrm{Sr})$, manganese $(\mathrm{Mn})$ thallium (TI), lead $(\mathrm{Pb})$, and uranium (U). Urine samples are diluted $1+9$ with $2 \%(v / v)$, double-distilled, concentrated nitric acid containing both iridium (Ir) and rhodium (Rh) for multi-internal standardization (more details via http://wwwn. cdc.gov/Nchs/Nhanes/2005-2006/UHM_D.htm). The test principle utilized high-performance liquid chromatographyelectrospray ionization-tandem mass spectrometry (HPLCESI-MS/MS) for the quantitative detection in urine of the following phthalate metabolites: monomethyl phthalate (MMP), monoethyl phthalate (MEP), monobutyl phthalate (MBP), monoisobutyl phthalate (MIBP), mono(3-carboxypropyl) phthalate (MCPP), mono(2-ethylhexyl) phthalate (MEHP), monobenzyl phthalate (MBzP), monoisononyl phthalate (MNP), mono(2-ethyl-5-oxohexyl) phthalate (MEOHP), mono(2-ethyl-5-hydroxyhexyl) phthalate (MEHHP), mono(2-ethyl-5-carboxypentyl) phthalate (MECPP), monocarboxyoctyl phthalate (MCOP), monocarboxynonyl phthalate (MCNP), and cyclohexane-1,2-dicarboxylic acidmono(hydroxy-isononyl) ester (MHNCH). Urine samples are processed using enzymatic deconjugation of the glucuronidated metabolites followed by on-line solid phase extraction (SPE) coupled with reversed phase HPLC-ESIMS/MS. Assay precision is improved by incorporating isotopically labeled internal standards of the phthalate metabolites and $\mathrm{MHNCH}$ (more details via http://wwwn.cdc. gov/Nchs/Nhanes/2005-2006/PHTHTE_D.htm).

Total and specific urine arsenic concentrations were determined by using inductively coupled-plasma dynamic reaction cell-mass spectrometry (ICP-DRC-MS) (more details via http://wwwn.cdc.gov/Nchs/Nhanes/2005-2006/UAS_D.htm) Urine is analyzed because urinary excretion is the major pathway for eliminating arsenic from the mammalian body. The method used on-line SPE coupled to HPLC and tandem mass spectrometry (HPL/CMS/MS). A sensitive method, SPE coupled on-line to HPLC and tandem mass spectrometry (MS/MS), was used for measuring bisphenol A (BPA), 4-tertoctylphenol (tOP), benzophenone-3 (BP-3), one chlorophenols triclosan, four parabens, and pesticides (more details via http://wwwn.cdc.gov/Nchs/Nhanes/2005-2006/EPH_D.htm and http://wwwn.cdc.gov/Nchs/Nhanes/2005-2006/PP_D. $\mathrm{htm})$. A quantitative procedure was used for the measurement of nitrate, perchlorate, and thiocyanate in human urine using ion chromatography coupled with electrospray tandem mass spectrometry. Chromatographic separation is achieved using an IonPac AS16 column with sodium hydroxide as the eluant. The eluant from the column is ionized using an electrospray interface to generate and transmit negative ions into the mass spectrometer (more details via http://wwwn.cdc. gov/Nchs/Nhanes/2005-2006/PTH_D.htm).

To detect and measure metabolites of polyaromatic hydrocarbons (more details via http://wwwn.cdc. gov/Nchs/Nhanes/2005-2006/PAH_D.htm), the procedure involved enzymatic hydrolysis of glucuronidated/sulfated $\mathrm{OH}$-polyaromatic hydrocarbons metabolites in urine, extraction, derivatization, and analysis using isotope dilution 
capillary gas chromatography tandem mass spectrometry (GC-MS/MS). Ion transitions specific to each analyte and carbon-13 labeled internal standards are monitored, and the abundances of each ion are measured. Moreover, solid phase extraction coupled to high-performance liquid chromatography-turbo ion spray ionization-tandem mass spectrometry (online SPE-HPLC-TIS-MS/MS) was used for the quantitative detection of perfluorooctane sulfonamide (PFOSA), 2-( $N$-methyl-perfluorooctane sulfonamido) acetic acid (Me-PFOSA-AcOH), 2-( $N$-ethyl-perfluorooctane sulfonamido) acetic acid (Et-PFOSA-AcOH), perfluorobutane sulfonate (PFBuS), perfluorohexane sulfonate (PFHxS), perfluorooctane sulfonate (PFOS), perfluoroheptanoate (PFHpA), perfluorooctanoate (PFOA), perfluorononanoate (PFNA), perfluorodecanoate (PFDeA), perfluoroundecanoate (PFUA), and perfluorododecanoate (PFDoA) (more details via http://wwwn.cdc. gov/Nchs/Nhanes/2005-2006/PFC_D.htm).

\section{Statistical analysis}

Americans aged $18-85$ with all the required study variables were included in the current statistical analysis.

Table 1 Characteristics of the study participants aged $18-85$ $(N=5563)$

\begin{tabular}{ll}
\hline & $N(\%)$ or mean \pm SD \\
\hline Age & $45.2 \pm 20.3$ \\
$18-39$ & $2507(45.1)$ \\
$40-59$ & $1486(26.7)$ \\
$60-85$ & $1570(28.2)$ \\
Sex & \\
Male & $2675(48.1)$ \\
Female & $2888(51.9)$ \\
Body mass index & $28.5 \pm 6.8$ \\
$<18.5$ & $101(1.9)$ \\
$18.5-24.9$ & $1620(30.9)$ \\
$25-29.9$ & $1734(33.1)$ \\
$30+$ & $1782(34.0)$ \\
Ratio of family income to poverty & \\
$0-4.9$ & $4318(82.0 \%)$ \\
$5+$ & $951(18.1 \%)$ \\
Serum cotinine (ng/mL) & $55.8 \pm 121.3$ \\
Serum vitamin D (ng/mL) & $21.3 \pm 9.4$ \\
Alcohol status & \\
$\geq 12$ drinks & $2994(68.8 \%)$ \\
Less than 12 drinks & $1355(31.2 \%)$ \\
Physical activity level & \\
Nongaging moderately & $3026(54.4 \%)$ \\
\hline & $2535(45.6 \%)$ \\
\hline &
\end{tabular}

Urinary environmental chemical concentrations were all $\log$ transformed because they were highly skewed to one side. Associations of urinary environmental chemical concentrations ( $x$ variables) and the five common sleep troubles in the past month ( $y$ variables; more details via http://wwwn.cdc.gov/Nchs/Nhanes/2005-2006/SLQ_D. $\mathrm{htm}$ ) were examined by using survey-weighted logistic regression models, with $P<0.05$ considered statistically significant. Covariates including urinary creatinine, age, sex, ratio of family income to poverty (proxy of socioeconomic status), body mass index, serum cotinine (biomarker of smoking status), vitamin D levels (more details via http://wwwn.cdc.gov/Nchs/Nhanes/2005-2006/VID_D. $\mathrm{htm}$ ), and physical activity level were adjusted. Statistical software STATA version 13.0 (STATA, College Station, Texas, USA; more details via http://www.stata.com/) was used to conduct all the analyses.

Table 2 Characteristics of sleep troubles in the study participants aged $18-85(N=5563)$

\begin{tabular}{ll}
\hline & Number $(\%)$ \\
\hline Wake-up at night & \\
Never & $2134(38.4)$ \\
1 time a month & $1091(19.6)$ \\
2-4 times a month & $1269(22.8)$ \\
5-15 times a month & $670(12.0)$ \\
16-30 times a month & $392(7.1)$ \\
Unrested during the day & \\
Never & $1746(31.4)$ \\
1 time a month & $893(16.1)$ \\
2-4 times a month & $1546(27.8)$ \\
5-15 times a month & $848(15.2)$ \\
16-30 times a month & $520(9.4)$ \\
Overly sleepy during the day \\
Never & $1908(34.3)$ \\
1 time a month & $1180(21.2)$ \\
2-4 times a month & $1450(26.1)$ \\
5-15 times a month & $663(11.9)$ \\
16-30 times a month & $352(6.3)$ \\
Leg jerks while sleeping & \\
Never & $4389(78.9)$ \\
1 time a month & $391(7.0)$ \\
2-4 times a month & $451(8.1)$ \\
5-15 times a month & $175(3.2)$ \\
$16-30$ times a month & $114(2.1)$ \\
Leg cramps while sleeping & \\
Never & $3833(68.9)$ \\
2-4 time a month & $663(11.9)$ \\
16-30 times a month & $103(1.9)$ \\
\hline
\end{tabular}


Table 3 Associations between urinary chemicals and wake-up at night in adults

\begin{tabular}{|c|c|c|c|c|}
\hline & $\begin{array}{l}\text { No wake-up at night } \\
(n=3225,58.1 \%)\end{array}$ & $\begin{array}{l}\text { Wake-up at night } \\
(n=2331,42.0 \%)\end{array}$ & OR $(95 \% \mathrm{CI})$ & $P$ value \\
\hline \multicolumn{5}{|l|}{ Arsenic $(n=1581, \mu \mathrm{g} / \mathrm{L})$} \\
\hline Total arsenic & $23.5 \pm 73.7$ & $22.2 \pm 51.9$ & $1.08(0.97-1.19)$ & 0.136 \\
\hline Arsenous acid & $0.9 \pm 0.7$ & $0.9 \pm 0.3$ & $0.79(0.31-2.01)$ & 0.607 \\
\hline Arsenic acid & $0.7 \pm 0.3$ & $0.8 \pm 0.5$ & $1.86(0.99-3.48)$ & 0.053 \\
\hline Arsenobetaine & $12.7 \pm 59.2$ & $12.4 \pm 41.8$ & $1.03(0.94-1.14)$ & 0.476 \\
\hline Arsenocholine & $0.4 \pm 0.3$ & $0.5 \pm 0.3$ & $1.05(0.68-1.62)$ & 0.805 \\
\hline Dimethylarsonic acid & $5.8 \pm 9.6$ & $5.7 \pm 7.9$ & $1.03(0.87-1.22)$ & 0.715 \\
\hline Monomethylarsonic acid & $1.0 \pm 1.1$ & $1.0 \pm 1.2$ & $1.08(0.74-1.56)$ & 0.681 \\
\hline Trimethylarsine oxide & $0.7 \pm 0.2$ & $0.7 \pm 0.2$ & $1.51(1.29-1.76)$ & $<0.001$ \\
\hline \multicolumn{5}{|l|}{ Phthalate $(n=1488, \mu \mathrm{g} / \mathrm{mL})$} \\
\hline Mono(carboxyoctyl) & $16.4 \pm 111.7$ & $16.9 \pm 73.2$ & $1.00(0.90-1.13)$ & 0.936 \\
\hline Mono(carboxynonyl) & $5.5 \pm 16.2$ & $6.7 \pm 31.1$ & $0.96(0.83-1.10)$ & 0.514 \\
\hline Mono-2-ethyl-5-carboxypentyl & $112.0 \pm 356.7$ & $93.9 \pm 235.2$ & $0.90(0.80-1.00)$ & 0.048 \\
\hline Mono- $n$-butyl & $48.4 \pm 338.5$ & $35.0 \pm 45.3$ & $0.97(0.85-1.10)$ & 0.625 \\
\hline Mono(3-carboxypropyl) & $4.3 \pm 15.7$ & $4.1 \pm 10.0$ & $1.01(0.91-1.13)$ & 0.813 \\
\hline Monoethyl & $433.8 \pm 1084.7$ & $508.1 \pm 1253.9$ & $1.04(0.94-1.14)$ & 0.432 \\
\hline Mono(2-ethyl-5-hydroxyhexyl) & $84.7 \pm 308.3$ & $75.0 \pm 211.9$ & $0.91(0.82-1.01)$ & 0.086 \\
\hline Mono(2-ethyl)-hexyl & $13.2 \pm 71.9$ & $10.0 \pm 31.4$ & $0.94(0.83-1.07)$ & 0.330 \\
\hline Monoisobutyl & $26.2 \pm 477.4$ & $11.1 \pm 19.9$ & $0.96(0.84-1.10)$ & 0.541 \\
\hline Mono- $n$-methyl & $3.5 \pm 12.9$ & $5.5 \pm 37.7$ & $1.05(0.95-1.16)$ & 0.290 \\
\hline Monoisononyl & $2.0 \pm 8.6$ & $1.8 \pm 5.5$ & $1.15(0.92-1.44)$ & 0.193 \\
\hline Mono(2-ethyl-5-oxohexyl) & $53.0 \pm 199.4$ & $45.7 \pm 124.5$ & $0.91(0.81-1.02)$ & 0.096 \\
\hline Monobenzyl & $35.4 \pm 542.2$ & $17.6 \pm 33.3$ & $0.96(0.85-1.09)$ & 0.508 \\
\hline Monocyclohexyl & $0.5 \pm 0.5$ & $0.5 \pm 1.1$ & $1.88(1.02-3.46)$ & 0.044 \\
\hline \multicolumn{5}{|c|}{ Polyfluoroalkyl compounds ( $n=1591, \mu \mathrm{g} / \mathrm{mL}$ ) } \\
\hline Perfluorooctanoic acid & $4.5 \pm 3.3$ & $4.5 \pm 3.7$ & $0.95(0.74-1.21)$ & 0.649 \\
\hline Perfluorooctane sulfonic acid & $21.0 \pm 15.9$ & $22.3 \pm 20.9$ & $0.99(0.83-1.20)$ & 0.947 \\
\hline Perfluorohexane sulfonic acid & $2.6 \pm 3.3$ & $2.4 \pm 2.6$ & $0.99(0.89-1.10)$ & 0.864 \\
\hline 2-(N-ethyl-PFOSA) acetate & $0.2 \pm 0.2$ & $0.2 \pm 0.1$ & $1.50(1.08-2.09)$ & 0.020 \\
\hline 2-( $N$-methyl-PFOSA) acetate & $0.6 \pm 0.8$ & $0.6 \pm 0.6$ & $1.08(0.96-1.22)$ & 0.183 \\
\hline Perfluorodecanoic acid & $0.5 \pm 0.6$ & $0.5 \pm 0.9$ & $0.98(0.83-1.17)$ & 0.838 \\
\hline Perfluorobutane sulfonic acid & $0.1 \pm 0.2$ & $0.1 \pm 0.1$ & $0.98(0.55-1.75)$ & 0.936 \\
\hline Perfluoroheptanoic acid & $0.4 \pm 0.4$ & $0.3 \pm 0.4$ & $0.99(0.82-1.19)$ & 0.887 \\
\hline Perfluorononanoic acid & $1.3 \pm 1.1$ & $1.4 \pm 1.4$ & $1.03(0.83-1.29)$ & 0.747 \\
\hline Perfluorooctane sulfonamide & $0.1 \pm 0.2$ & $0.1 \pm 0.1$ & $1.09(0.88-1.36)$ & 0.389 \\
\hline Perfluoroundecanoic acid & $0.3 \pm 0.5$ & $0.3 \pm 0.6$ & $0.99(0.82-1.19)$ & 0.908 \\
\hline Perfluorododecanoic acid & $0.1 \pm 0.1$ & $0.1 \pm 0.1$ & $1.72(1.08-2.73)$ & 0.026 \\
\hline
\end{tabular}

Adjusted for age, sex, body mass index, ratio of family income to poverty, serum cotinine (smoking status), vitamin D level, physical activity level, urinary creatinine and subsample weighting

\section{Ethics concerns}

Since there are only secondary data analyses employed without any participant personal information identified by extracting statistical data from the NHANES website in the present study, no further ethics approval for conducting the present study is required (more details via http://www. ethicsguidebook.ac.uk/Secondary-analysis-106).

\section{Results}

Table 1 shows characteristics of the included study participants. Of all 5563 Americans aged 18-85, 2331 (42.0\%) had wake-up at night, 2914 (52.5\%) felt unrested during the day, $740(13.4 \%)$ had leg jerks while sleeping, and 1059 (19.1\%) had leg cramps while sleeping for $2+$ times a month. Table 2 lists the frequency of common sleep troubles in the 
Table 4 Associations between urinary chemicals and unrested during the day in adults

\begin{tabular}{lllc}
\hline & $\begin{array}{l}\text { No unrested } \\
(n=2639,47.5 \%)\end{array}$ & $\begin{array}{l}\text { Unrested during the day } \\
(n=2914,52.5 \%)\end{array}$ & OR (95\% CI) \\
\hline Phenol $(n=1486, \mathrm{ng} / \mathrm{mL})$ & & & $1.93(1.02-3.67)$ \\
$\quad$ 4-Tert-octylphenol & $0.2 \pm 0.3$ & $0.2 \pm 0.1$ & 0.044 \\
Polyfluoroalkyl compounds $(n=1592, \mu \mathrm{g} / \mathrm{mL})$ & & $1.01(0.84-1.22)$ \\
Perfluorooctanoic acid & $4.6 \pm 3.3$ & $4.5 \pm 3.6$ & $1.09(0.93-1.28)$ \\
Perfluorooctane sulfonic acid & $22.4 \pm 19.5$ & $20.7 \pm 16.9$ & $1.03(0.94-1.14)$ \\
Perfluorohexane sulfonic acid & $2.5 \pm 2.9$ & $2.5 \pm 3.2$ & $1.29(0.82-2.00)$ \\
2-(N-ethyl-PFOSA) acetate & $0.2 \pm 0.1$ & $0.2 \pm 0.3$ & $1.24(1.02-1.51)$ \\
2-(N-methyl-PFOSA) acetate & $0.6 \pm 0.7$ & $0.6 \pm 0.7$ & $1.10(0.92-1.30)$ \\
Perfluorodecanoic acid & $0.5 \pm 0.9$ & $0.5 \pm 0.6$ & $1.42(1.02-1.98)$ \\
Perfluorobutane sulfonic acid & $0.1 \pm 0.1$ & $0.1 \pm 0.2$ & 0.4678 \\
Perfluoroheptanoic acid & $0.3 \pm 0.3$ & $0.4 \pm 0.5$ & 0.248 \\
Perfluorononanoic acid & $1.4 \pm 1.3$ & $1.4 \pm 1.2$ & 0.035 \\
Perfluorooctane sulfonamide & $0.1 \pm 0.1$ & $0.1 \pm 0.2$ & $1.14(0.95-1.38)$ \\
Perfluoroundecanoic acid & $0.3 \pm 0.7$ & $0.3 \pm 0.4$ & $1.15(0.93-1.43)$ \\
Perfluorododecanoic acid & $0.1 \pm 0.04$ & $0.2 \pm 0.1$ & $1.08(0.85-1.36)$ \\
\hline
\end{tabular}

Adjusted for age, sex, body mass index, ratio of family income to poverty, serum cotinine (smoking status), vitamin D level, physical activity level, urinary creatinine and sub-sample weighting

study participants. Tables $3,4,5$, and 6 present the associations between urinary chemical concentrations and common sleep troubles, except for feeling overly sleeping during the day with no association found, accordingly. To be specific, higher levels of urinary arsenic, phthalates, and polyfluoroalkyl compounds were associated with wake-up at night. Higher levels of urinary 4-tert-octylphenol and polyfluoroalkyl compounds were associated with being unrested during the day. Higher levels of urinary arsenic, polyaromatic hydrocarbons, and polyfluoroalkyl compounds were associated with leg jerks while sleeping. Higher levels of urinary pesticides, heavy metals, phthalates, and polyaromatic hydrocarbons were associated with leg cramps while sleeping. There were no significant associations with other environmental chemicals such as parabens, bisphenol A, benzophenone-3, triclosan, perchlorate, nitrate, or thiocyanate (data not shown).

\section{Discussion}

\section{Arsenic and sleep}

Low level of arsenic exposure was previously observed to be associated with sleep disturbance in copper smelter workers ( $n=680$; Lilis et al. 1985). Long-term poisoning was also found to affect sleep disorder in children (Ishi and Tamaoka 2015). In the present study, higher levels of urinary arsenic were found to be associated with wake-up at night and leg jerk while sleeping. However, to date no experimental research was available to confirm the biological mechanism yet.

\section{Pesticides and sleep}

Pesticide exposure, particularly 2,4,5-trichlorophenol, was recently found to influence idiopathic REM sleep behavior disorder in older adults ( $n=694$; Postuma et al. 2012; Neuberger et al. 1998). In the limited experimental research, exposure to pesticides seemed to reduce sleep time in male mice (21-24 g; Chaturvedi 1993). In the present study using national representative human sample, 2,5-dichlorophenol and 2,4-dichlorophenol showed borderline associations with leg cramps in sleeping. This would need future longitudinal or experimental research to confirm or refute the findings in order to rule out the statistical significance by chance.

\section{Heavy metals and sleep}

Uranium might directly affect the brain. Previously, it was observed that chronic uranium exposure $(40 \mathrm{mg} 1(-1)$ in drinking water, for 90 days) increased in rapid eye movement sleep in rats (Lestaevel et al. 2005). In the present study, the consistent finding was shown in higher levels of urinary uranium and urinary antimony and leg cramps in sleeping.

\section{Phthalates and sleep}

The intravenous or intraperitoneal administration of bis(2ethylhexyl) phthalate could prolong hexobarbital sleep time in mice and rats by enlarging the lipophilic pool (Swinyard et al. 1976). In the present study, monocyclohexyl showed an association with wake-up at night, and mono(carboxynonyl) 
Table 5 Associations between urinary chemicals and leg jerks while sleeping in adults

\begin{tabular}{|c|c|c|c|c|}
\hline & $\begin{array}{l}\text { No leg jerks } \\
(n=4780,86.6 \%)\end{array}$ & $\begin{array}{l}\text { Leg jerks while sleeping } \\
(n=740,13.4 \%)\end{array}$ & OR $(95 \% \mathrm{CI})$ & $P$ value \\
\hline \multicolumn{5}{|l|}{ Arsenic $(n=1567, \mu \mathrm{g} / \mathrm{L})$} \\
\hline Total arsenic & $22.1 \pm 56.7$ & $28.8 \pm 106.5$ & $1.03(0.88-1.21)$ & 0.714 \\
\hline Arsenous acid & $0.9 \pm 0.6$ & $0.9 \pm 0.2$ & $0.89(0.32-2.45)$ & 0.802 \\
\hline Arsenic acid & $0.8 \pm 0.4$ & $0.8 \pm 0.6$ & $0.73(0.28-1.94)$ & 0.508 \\
\hline Arsenobetaine & $11.7 \pm 43.4$ & $18.5 \pm 92.2$ & $0.99(0.87-1.13)$ & 0.869 \\
\hline Arsenocholine & $0.4 \pm 0.3$ & $0.5 \pm 0.3$ & $1.02(0.60-1.70)$ & 0.951 \\
\hline Dimethylarsonic acid & $5.8 \pm 9.5$ & $5.2 \pm 4.7$ & $0.96(0.80-1.14)$ & 0.586 \\
\hline Monomethylarsonic acid & $1.0 \pm 1.2$ & $0.9 \pm 0.6$ & $0.97(0.60-1.58)$ & 0.904 \\
\hline Trimethylarsine oxide & $0.7 \pm 0.2$ & $0.7 \pm 0.4$ & $1.72(1.09-2.72)$ & 0.022 \\
\hline \multicolumn{5}{|c|}{ Polyaromatic hydrocarbons $(n=1412, \mathrm{ng} / \mathrm{L})$} \\
\hline 2-Hydroxyfluorene & $722.2 \pm 1257.0$ & $912.4 \pm 1540.5$ & $1.21(1.02-1.43)$ & 0.029 \\
\hline 3-Hydroxyfluorene & $353.8 \pm 880.4$ & $461.8 \pm 784.5$ & $1.19(1.02-1.39)$ & 0.029 \\
\hline 9-Hydroxyfluorene & $649.8 \pm 1237.2$ & $703.3 \pm 1243.4$ & $1.21(0.96-1.53)$ & 0.104 \\
\hline 1-Hydroxyphenanthrene & $265.6 \pm 528.0$ & $259.6 \pm 305.9$ & $1.23(0.97-1.55)$ & 0.081 \\
\hline 2-Hydroxyphenanthrene & $123.4 \pm 279.5$ & $127.3 \pm 174.7$ & $1.17(0.91-1.51)$ & 0.207 \\
\hline 3-Hydroxyphenanthrene & $199.0 \pm 486.5$ & $223.6 \pm 370.5$ & $1.22(0.95-1.56)$ & 0.114 \\
\hline 1-Hydroxypyrene & $214.0 \pm 627.3$ & $279.9 \pm 790.4$ & $1.22(1.03-1.45)$ & 0.025 \\
\hline 1-Hydroxynapthalene (1-naphthol) & $22,657.5 \pm 177,331.5$ & $175,350.4 \pm 1,968,678.0$ & $1.05(0.91-1.21)$ & 0.497 \\
\hline 2-Hydroxynapthalene (2-naphthol) & $9312.6 \pm 25,015.5$ & $10,078.5 \pm 14,846.3$ & $1.02(0.80-1.30)$ & 0.849 \\
\hline 4-Hydroxyphenanthrene & $54.9 \pm 99.4$ & $50.6 \pm 52.5$ & $1.06(0.85-1.33)$ & 0.575 \\
\hline \multicolumn{5}{|c|}{ Polyfluoroalkyl compounds ( $n=1584, \mu \mathrm{g} / \mathrm{mL}$ ) } \\
\hline Perfluorooctanoic acid & $4.5 \pm 3.3$ & $4.7 \pm 4.1$ & $1.05(0.89-1.24)$ & 0.523 \\
\hline Perfluorooctane sulfonic acid & $21.4 \pm 18.0$ & $22.2 \pm 19.0$ & $1.05(0.90-1.24)$ & 0.488 \\
\hline Perfluorohexane sulfonic acid & $2.5 \pm 3.0$ & $2.8 \pm 3.1$ & $1.06(0.87-1.31)$ & 0.528 \\
\hline 2-(N-ethyl-PFOSA) acetate & $0.2 \pm 0.2$ & $0.2 \pm 0.1$ & $0.94(0.47-1.91)$ & 0.867 \\
\hline 2-( $N$-methyl-PFOSA) acetate & $0.6 \pm 0.7$ & $0.6 \pm 0.5$ & $1.27(1.12-1.44)$ & 0.001 \\
\hline Perfluorodecanoic acid & $0.5 \pm 0.7$ & $0.6 \pm 0.9$ & $1.03(0.84-1.27)$ & 0.744 \\
\hline Perfluorobutane sulfonic acid & $0.1 \pm 0.2$ & $0.1 \pm 0.1$ & $1.65(1.03-2.66)$ & 0.040 \\
\hline Perfluoroheptanoic acid & $0.3 \pm 0.4$ & $0.4 \pm 0.7$ & $1.26(1.02-1.56)$ & 0.035 \\
\hline Perfluorononanoic acid & $1.4 \pm 1.3$ & $1.3 \pm 1.2$ & $1.05(0.82-1.33)$ & 0.694 \\
\hline Perfluorooctane sulfonamide & $0.1 \pm 0.2$ & $0.1 \pm 0.1$ & $0.89(0.66-1.21)$ & 0.448 \\
\hline Perfluoroundecanoic acid & $0.3 \pm 0.5$ & $0.3 \pm 0.6$ & $0.97(0.77-1.21)$ & 0.746 \\
\hline Perfluorododecanoic acid & $0.1 \pm 0.1$ & $0.1 \pm 0.1$ & $1.39(0.55-3.52)$ & 0.466 \\
\hline
\end{tabular}

Adjusted for age, sex, body mass index, ratio of family income to poverty, serum cotinine (smoking status), vitamin D level, physical activity level, urinary creatinine and sub-sample weighting

and mono(3-carboxypropyl) showed associations with leg cramps in sleeping. These would also need future longitudinal or experimental research to confirm or refute the findings in order to rule out the statistical significance by chance.

\section{Polyaromatic hydrocarbons and sleep}

There was no direct research evidence on the relationship of polyaromatic hydrocarbons and sleep health. In the present study, it is the first time to show that higher urinary polyaromatic hydrocarbons, such as 2-hydroxyfluorene, 9-hydroxyfluorene, 2-hydroxyphenanthrene, and 1-hydroxypyrene, were significantly associated with leg cramps in sleeping. Similarly, urinary 2-hydroxyfluorene, 3-hydroxyfluorene, 1hydroxypyrene were significantly associated with leg jerks in sleeping. Levels of other known urinary polyaromatic hydrocarbons were also higher in people with sleep disturbances, but the statistical significance was not reached.

\section{Polyfluoroalkyl compounds and sleep}

There were no indications that there could be any association between polyfluoroalkyl compounds and sleep health in the past. Polyfluoroalkyl compounds in human 
Table 6 Associations between urinary chemicals and leg cramps while sleeping in adults

\begin{tabular}{|c|c|c|c|c|}
\hline & $\begin{array}{l}\text { No leg cramps } \\
(n=4496,80.9 \%)\end{array}$ & $\begin{array}{l}\text { Leg cramps while sleeping } \\
(n=1059,19.1 \%)\end{array}$ & OR $(95 \% \mathrm{CI})$ & $P$ value \\
\hline \multicolumn{5}{|l|}{ Pesticides $(n=1489, \mu \mathrm{g} / \mathrm{L})$} \\
\hline 2,5-dichlorophenol & $228.2 \pm 1242.6$ & $175.0 \pm 772.2$ & $1.12(1.02-1.23)$ & 0.018 \\
\hline$O$-phenyl phenol & $0.2 \pm 0.6$ & $0.3 \pm 2.2$ & $0.98(0.72-1.35)$ & 0.904 \\
\hline 2,4-Dichlorophenol & $7.4 \pm 45.9$ & $5.5 \pm 24.0$ & $1.17(1.03-1.32)$ & 0.017 \\
\hline 2,4,5-Trichlorophenol & $0.1 \pm 0.3$ & $0.1 \pm 0.1$ & $1.08(0.86-1.37)$ & 0.466 \\
\hline 2,4,6-Trichlorophenol & $0.7 \pm 2.7$ & $0.7 \pm 2.4$ & $0.91(0.67-1.23)$ & 0.511 \\
\hline \multicolumn{5}{|l|}{ Heavy metals $(n=1576, \mu \mathrm{g} / \mathrm{L})$} \\
\hline Barium & $2.3 \pm 3.9$ & $2.2 \pm 3.2$ & $0.93(0.81-1.06)$ & 0.257 \\
\hline Cadmium & $0.4 \pm 0.4$ & $0.5 \pm 0.5$ & $1.05(0.91-1.21)$ & 0.463 \\
\hline Cobalt & $0.5 \pm 1.2$ & $0.8 \pm 2.3$ & $1.07(0.87-1.30)$ & 0.506 \\
\hline Cesium & $2.3 \pm 3.9$ & $2.2 \pm 3.2$ & $0.92(0.78-1.08)$ & 0.278 \\
\hline Molybdenum & $5.7 \pm 4.0$ & $5.4 \pm 4.5$ & $0.98(0.79-1.20)$ & 0.813 \\
\hline Lead & $61.1 \pm 53.1$ & $57.7 \pm 51.1$ & $1.02(0.80-1.29)$ & 0.895 \\
\hline Antimony & $1.0 \pm 2.3$ & $0.9 \pm 0.8$ & $1.22(1.05-1.43)$ & 0.015 \\
\hline Thallium & $0.2 \pm 1.3$ & $0.1 \pm 0.2$ & $0.87(0.72-1.05)$ & 0.136 \\
\hline Tungsten & $0.2 \pm 0.1$ & $0.2 \pm 0.1$ & $1.07(0.91-1.26)$ & 0.392 \\
\hline Uranium & $0.1 \pm 0.3$ & $0.2 \pm 0.4$ & $1.27(1.06-1.51)$ & 0.011 \\
\hline \multicolumn{5}{|c|}{ Polyaromatic hydrocarbons $(n=1425, \mathrm{ng} / \mathrm{L})$} \\
\hline 2-Hydroxyfluorene & $738.5 \pm 1275.0$ & $764.3 \pm 1373.7$ & $1.19(1.00-1.43)$ & 0.054 \\
\hline 3-Hydroxyfluorene & $371.7 \pm 904.2$ & $340.3 \pm 676.4$ & $1.14(0.96-1.36)$ & 0.121 \\
\hline 9-Hydroxyfluorene & $644.6 \pm 1212.2$ & $692.2 \pm 1320.2$ & $1.28(1.12-1.45)$ & 0.001 \\
\hline 1-Hydroxyphenanthrene & $259.3 \pm 520.7$ & $284.1 \pm 419.3$ & $1.21(0.98-1.50)$ & 0.074 \\
\hline 2-Hydroxyphenanthrene & $121.5 \pm 281.9$ & $131.4 \pm 191.6$ & $1.24(1.00-1.53)$ & 0.046 \\
\hline 3-Hydroxyphenanthrene & $203.7 \pm 499.2$ & $189.1 \pm 322.1$ & $1.18(0.97-1.43)$ & 0.086 \\
\hline 1-Hydroxypyrene & $215.3 \pm 639.9$ & $245.8 \pm 676.3$ & $1.28(1.10-1.50)$ & 0.004 \\
\hline 1-Hydroxynapthalene (1-naphthol) & $23,290.6 \pm 182,622.3$ & $124,604.9 \pm 1,616,734.0$ & $1.05(0.94-1.16)$ & 0.368 \\
\hline 2-Hydroxynapthalene (2-naphthol) & $8859.9 \pm 20,058.9$ & $11,969.1 \pm 36,521.9$ & $1.14(0.97-1.33)$ & 0.104 \\
\hline 4-Hydroxyphenanthrene & $53.8 \pm 97.5$ & $55.6 \pm 80.6$ & $1.15(0.92-1.43)$ & 0.214 \\
\hline \multicolumn{5}{|l|}{ Phthalate $(n=1488, \mu \mathrm{g} / \mathrm{mL})$} \\
\hline Mono(carboxyoctyl) & $17.8 \pm 106.8$ & $11.5 \pm 20.3$ & $1.10(0.95-1.28)$ & 0.174 \\
\hline Mono(carboxynonyl) & $6.2 \pm 25.8$ & $5.3 \pm 12.5$ & $1.15(1.02-1.31)$ & 0.025 \\
\hline Mono-2-ethyl-5-carboxypentyl & $103.8 \pm 313.6$ & $105.2 \pm 291.9$ & $1.06(0.90-1.24)$ & 0.489 \\
\hline Mono- $n$-butyl & $41.7 \pm 272.7$ & $46.1 \pm 162.8$ & $1.13(0.97-1.31)$ & 0.100 \\
\hline Mono(3-carboxypropyl) & $4.3 \pm 14.7$ & $3.9 \pm 6.0$ & $1.20(1.02-1.42)$ & 0.032 \\
\hline Monoethyl & $480.7 \pm 1212.1$ & $404.0 \pm 912.0$ & $1.06(0.98-1.14)$ & 0.141 \\
\hline Mono(2-ethyl-5-hydroxyhexyl) & $81.6 \pm 280.8$ & $75.6 \pm 219.3$ & $1.06(0.92-1.21)$ & 0.412 \\
\hline Mono(2-ethyl)-hexyl & $12.4 \pm 62.8$ & $9.2 \pm 26.6$ & $1.06(0.93-1.21)$ & 0.360 \\
\hline Monoisobutyl & $21.4 \pm 397.3$ & $11.7 \pm 23.5$ & $1.12(1.00-1.26)$ & 0.057 \\
\hline Mono- $n$-methyl & $4.3 \pm 27.5$ & $4.9 \pm 23.5$ & $1.03(0.87-1.21)$ & 0.735 \\
\hline Monoisononyl & $2.1 \pm 8.2$ & $1.2 \pm 1.3$ & $1.07(0.81-1.43)$ & 0.605 \\
\hline Mono(2-ethyl-5-oxohexyl) & $50.5 \pm 177.8$ & $46.9 \pm 136.1$ & $1.04(0.90-1.19)$ & 0.587 \\
\hline Monobenzyl & $29.3 \pm 451.3$ & $20.3 \pm 45.1$ & $1.06(0.93-1.19)$ & 0.357 \\
\hline Monocyclohexyl & $0.5 \pm 0.5$ & $0.5 \pm 1.7$ & $1.38(0.67-2.85)$ & 0.353 \\
\hline
\end{tabular}

Adjusted for age, sex, body mass index, ratio of family income to poverty, serum cotinine (smoking status), vitamin D level, physical activity level, urinary creatinine and subsample weighting

health is an under-studied area, mostly with a focus on fetal growth, neuro-behavioral problems in infants or children, or cognitive function in older adults (Bach et al. 2015; Donauer et al. 2015; Power et al. 2013; 
Maisonet et al. 2012; Hoffman et al. 2010). In the present study using national representative human sample, the new observations have consistently shown higher levels of urinary 2-( $N$-ethyl-PFOSA) acetate and perfluorododecanoic acid in people with wake-up at night, higher levels of urinary 2-( $N$-methyl-PFOSA) acetate, perfluorobutane sulfonic acid in people feeling unrested during the day, and higher levels of 2-( $N$-methylPFOSA) acetate, perfluorobutane sulfonic acid and perfluoroheptanoic acid in people with leg jerks in sleeping. However, to date there has been no experimental research to confirm yet.

\section{Strengths and limitations}

The present study has a few strengths. Firstly, this study was conducted in a large and nationally representative human sample with mixed ethnicities and socioeconomic status. Secondly, this was the first time to examine the risk associations between different sets of environmental chemical concentrations and common sleep troubles. However, there are still some limitations that cannot be ignored. First, there could be still other emerging chemicals from the living environments that we might not yet know and would need future research to further identify and examine. Second, objective measurements on sleep patterns were not available. Third, the causality cannot be established in the present study due to the crosssectional study design in nature. Therefore, future studies with longitudinal or experimental study designs to confirm or refute the current findings and, if at all, to understand the persisting risk effects along the life course from those mentioned above environmental chemicals would be warranted.

\section{Directions for future research, practice, and policy}

Urinary arsenic, pesticides, heavy metals, phthalates, polyaromatic hydrocarbons and polyfluoroalkyl compounds were associated with common sleep troubles in adults For future research, studies understanding the biological mechanism and monitoring the risk effects along the life course with a longitudinal or experimental approach would be needed. For practice and policy, elimination of these environmental chemicals in order to improve sleep health might be considered.

\section{Acknowledgements None}

\section{Compliance with ethical standards}

Conflict of interest The author declares that she has no conflict of interest.
Open Access This article is distributed under the terms of the Creative Commons Attribution 4.0 International License (http:// creativecommons.org/licenses/by/4.0/), which permits unrestricted use, distribution, and reproduction in any medium, provided you give appropriate credit to the original author(s) and the source, provide a link to the Creative Commons license, and indicate if changes were made.

\section{References}

Bach CC, Bech BH, Brix N, Nohr EA, Bonde JP, Henriksen TB (2015) Perfluoroalkyl and polyfluoroalkyl substances and human fetal growth: a systematic review. Crit Rev Toxicol 45:53-67

Chaturvedi AK (1993) Toxicological evaluation of mixtures of ten widely used pesticides. J Appl Toxicol 13:183-188

Donauer S, Chen A, Xu Y, Calafat AM, Sjodin A, Yolton K (2015) Prenatal exposure to polybrominated diphenyl ethers and polyfluoroalkyl chemicals and infant neurobehavior. J Pediatr 166:736-742

Hoffman K, Webster TF, Weisskopf MG, Weinberg J, Vieira VM (2010) Exposure to polyfluoroalkyl chemicals and attention deficit/ hyperactivity disorder in U.S. children 12-15 years of age. Environ Health Perspect 118:1762-1767

Ishi K, Tamaoka A (2015) Ten-years records of organic arsenic (diphenylarsinic acid) poisoning: epidemiology, clinical feature, metabolism, and toxicity. Brain Nerve 67:5-18

Lestaevel P, Bussy C, Paquet F, Dhieux B, Clarençon D, Houpert P, Gourmelon P (2005) Changes in sleep-wake cycle after chronic exposure to uranium in rats. Neurotoxicol Teratol 27:835-840

Lilis R, Valciukas JA, Weber JP, Malkin J (1985) Effects of low-level lead and arsenic exposure on copper smelter workers. Arch Environ Health 40:38-47

Maisonet M, Terrell ML, McGeehin MA, Christensen KY, Holmes A, Calafat AM, Marcus M (2012) Maternal concentrations of polyfluoroalkyl compounds during pregnancy and fetal and postnatal growth in British girls. Environ Health Perspect 120:1432-1437

McCarty DE, Chesson AL Jr, Jain SK, Marino AA (2014) The link between vitamin D metabolism and sleep medicine. Sleep Med Rev 18:311-319

Mulligan KJ, Davidson TM, Caruso JA (1990) Feasibility of the direct analysis of urine by inductively coupled argon plasma mass-spectrometry for biological monitoring of exposure to metals. J Anal At Spectrom 5(4):301-306

Neuberger M, Kundi M, Jäger R (1998) Chloracne and morbidity after dioxin exposure (preliminary results). Toxicol Lett 96-97:347-350

Postuma RB, Montplaisir JY, Pelletier A, Dauvilliers Y, Oertel W, Iranzo A, Ferini-Strambi L, Arnulf I, Hogl B, Manni R, Miyamoto T, Mayer G, Stiasny-Kolster K, Puligheddu M, Ju Y, Jennum P, Sonka K, Santamaria J, Fantini ML, Zucconi M, Leu-Semenescu S, Frauscher B, Terzaghi M, Miyamoto M, Unger MM, Cochen De Cock V, Wolfson C (2012) Environmental risk factors for REM sleep behavior disorder: a multicenter case-control study. Neurology 79:428-434

Power MC, Webster TF, Baccarelli AA, Weisskopf MG (2013) Crosssectional association between polyfluoroalkyl chemicals and cognitive limitation in the National Health and Nutrition Examination Survey. Neuroepidemiology 40:125-132

Shiue I (2015a) Are urinary polyaromatic hydrocarbons associated with adult hypertension, heart attack, and cancer? USA NHANES, 20112012. Environ Sci Pollut Res Int. doi:10.1007/s11356-015-4922-8

Shiue I (2015b) Urinary heavy metals, phthalates, phenols, thiocyanate, parabens, pesticides, polyaromatic hydrocarbons but not arsenic or polyfluorinated compounds are associated with adult oral health: USA NHANES, 2011-2012. Environ Sci Pollut Res Int. doi:10.1007/s11356-015-4561-0 
Shiue I (2015c) Urinary parabens and polyaromatic hydrocarbons independent of health conditions are associated with adult emotional support needs: USA NHANES, 2005-2008. Environ Sci Pollut Res Int. doi:10.1007/s11356-015-4749-3

Shiue I (2015d) Urinary heavy metals, phthalates and polyaromatic hydrocarbons independent of health events are associated with adult depression: USA NHANES, 2011-2012. Environ Sci Pollut Res Int. doi:10.1007/s11356-015-4944-2

Shiue I (2014a) Arsenic, heavy metals, phthalates, pesticides, hydrocarbons and polyfluorinated compounds but not parabens or phenols are associated with adult remembering condition: US NHANES, 2011-2012. Environ Sci Pollut Res Int 22:6381-6386

Shiue I (2014b) Higher urinary heavy metal, phthalate, and arsenic but not parabens concentrations in people with high blood pressure, U.S. NHANES, 2011-2012. Int J Environ Res Public Health 11:5989-5899

Shiue I (2013a) Urine phthalate concentrations are higher in people with stroke: United States National Health and Nutrition Examination Surveys (NHANES), 2001-2004. Eur J Neurol 20:728-731
Shiue I (2013b) Association of urinary arsenic, heavy metal, and phthalate concentrations with food allergy in adults: National Health and Nutrition Examination Survey, 2005-2006. Ann Allergy Asthma Immunol 111:421-423

Shiue I (2013c) Urinary environmental chemical concentrations and vitamin D are associated with vision, hearing, and balance disorders in the elderly. Environ Int 53:41-46

Shiue I (2013d) Low vitamin D levels in adults with longer time to fall asleep: US NHANES, 2005-2006. Int J Cardiol 168: 5074-5075

Swinyard EA, Woodhead JH, Petersen RV (1976) Nonspecific effect of bis(2-ethylhexyl) phthalate on hexobarbital sleep time. J Pharm Sci 65:733-734

Zeitlhofer J, Schmeiser-Rieder A, Tribl G, Rosenberger A, Bolitschek J, Kapfhammer G, Saletu B, Katschnig H, Holzinger B, Popovic R, Kunze M (2000) Sleep and quality of life in the Austrian population. Acta Neurol Scand 102:249-257 